\title{
Optimal Convergence Rate of $\theta$-Maruyama Method for Stochastic Volterra Integro-Differential Equations with Riemann-Liouville Fractional Brownian Motion
}

\author{
Mengjie Wang, Xinjie Dai and Aiguo Xiao* \\ School of Mathematics and Computational Science E Hunan Key Laboratory for \\ Computation and Simulation in Science and Engineering, Xiangtan University, \\ Xiangtan, Hunan 411105, China
}

Received 14 December 2020; Accepted (in revised version) 16 March 2021

\begin{abstract}
This paper mainly considers the optimal convergence analysis of the $\theta-$ Maruyama method for stochastic Volterra integro-differential equations (SVIDEs) driven by Riemann-Liouville fractional Brownian motion under the global Lipschitz and linear growth conditions. Firstly, based on the contraction mapping principle, we prove the well-posedness of the analytical solutions of the SVIDEs. Secondly, we show that the $\theta$-Maruyama method for the SVIDEs can achieve strong first-order convergence. In particular, when the $\theta$-Maruyama method degenerates to the explicit EulerMaruyama method, our result improves the conclusion that the convergence rate is $H+\frac{1}{2}, H \in\left(0, \frac{1}{2}\right)$ by Yang et al., J. Comput. Appl. Math., 383 (2021), 113156. Finally, the numerical experiment verifies our theoretical results.
\end{abstract}

AMS subject classifications: 65C30, 65C20, 65L20

Key words: Stochastic Volterra integro-differential equations, Riemann-Liouville fractional Brownian motion, well-posedness, strong convergence.

\section{Introduction}

Volterra integro-differential equations play an important role in biology, physics and engineering [1-4] and other aspects, especially in the study of heat conduction [3]. With the continuous development of science and technology [5-9], researchers have put forward many questions about Volterra integro-differential equations from practical problems. In 1966, Barnes and Allan [10] gave a simple definition of fractional Brownian motion based

*Corresponding author.

Email: xag@xtu.edu.cn (A. Xiao) 
on the Riemann-Liouville integral, then the fractional Brownian motion gradually attracted much attention. The fractional Brownian motion of the Riemann-Liouville type was written by

$$
B_{H}(t)=\frac{1}{\Gamma\left(H+\frac{1}{2}\right)} \int_{0}^{t}(t-s)^{H-\frac{1}{2}} d B(s), \quad t \geq 0,
$$

where $\Gamma(\cdot)$ is a Gamma function, $H \in(0,1), \mathrm{B}(\mathrm{s})$ is an $m$-dimensional standard Wiener process defined on the complete probability space $\left(\Omega, \mathcal{F}_{t},\left\{\mathcal{F}_{t}\right\}_{t \geq 0}, P\right)$. When $H=1 / 2, B_{H}(t)$ degenerates into the standard Brownian motion; When $H \in\left(0, \frac{1}{2}\right), B_{H}(t)$ is not a semimartingale, and the increment is relevant due to singularity [11-13]. These properties of fractional Brownian motion bring about widespread attention, and fractional Brownian motion is used in physics, statistics, engineering, options [14-16] in the following decades. In fact, differential equations driven by fractional Brownian motion have become important mathematical models including Cox-Ingersoll-Ross model, etc. [12,1721]. Therefore, Volterra integro-differential equations with fractional Brownian motion have great research significance.

This paper mainly considers the nonlinear singular stochastic Volterra integrodifferential equations (SVIDEs)

$$
\left\{\begin{array}{l}
\frac{d x(t)}{d t}=f(x(t))+\int_{0}^{t}(t-\tau)^{H-\frac{1}{2}} g(x(\tau)) d B(\tau), \quad t \in[0, T] \\
x(0)=x_{0}
\end{array}\right.
$$

where $f: \mathbb{R}^{d} \rightarrow \mathbb{R}^{d}, g: \mathbb{R}^{d} \rightarrow \mathbb{R}^{d \times m}$ are Borel measurable real-valued functions, $H \in\left(0, \frac{1}{2}\right)$. Yang et al. [19] firstly considered the linear case of SVIDEs (1.1) and gave the strong convergence order of the Euler-Maruyama (EM) method, which is $\min \left\{H+\frac{1}{2}, 1\right\} \quad(0<$ $H<1)$. Based on [19], the purpose of this paper is as follows:

- Because the well-posedness of SVIDEs (1.1) was left over from literature [19], this paper firstly proves that (1.1) has a unique strong solution. The tool used in the proof is the contraction mapping principle [22-25].

- We investigate the strong convergence order of the $\theta$-Maruyama method, which improves the corresponding result in [19].

In fact, some progresses have been made in the strong convergence order of numerical methods for other classes of SVIDEs [26-29].

As shown in Section 2, (1.1) can be rewritten as the stochastic Volterra integral equations (SVIEs)

$$
x(t)=x(0)+\int_{0}^{t} f(x(s)) d s+\int_{0}^{t} \frac{1}{H+\frac{1}{2}}(t-s)^{H+\frac{1}{2}} g(x(s)) d B(s),
$$

where $t \in[0, T]$. It is worth emphasizing that the kernel function of (1.2) is not Lipschitz continuous, but Hölder continuous with index $H+\frac{1}{2}, H \in\left(0, \frac{1}{2}\right)$. Indeed, for the strong 
convergence order of numerical methods for SVIEs, some interesting conclusions also have been obtained. For the linear SVIEs with convolution kernels, Liang et al. [30] obtained the superconvergence order of EM method when the kernel function is Lipschitz continuous and satisfies an additional assumption. Moreover, the related conclusions for the other classes of SVIEs by using Euler-type method can be obtained [20,31,32]. Similarly, if we add a jump term to the right side of the SVIEs, Khalaf et al. [33] showed that the strong convergence order can reach up to order 1 if the kernel function is Lipschitz continuous and the diffusion coeffcient and the jump coeffcient satisfy a same additional assumption as in [30]. For the SVIEs with doubly singular kernels, Dai and Xiao [34] analysed the strong convergence order of EM method, and constructed the fast EM method to improve the computational effciency. In addition, Li et al. [36] also discussed asymptotic sparation for SVIEs with doubly singular kernels, which extends the corresponding result of [35].

In order to solve numerically the nonlinear SVIEs (1.2), this article considers the $\theta-$ Maruyama method

$$
Y_{n}=x_{0}+h \sum_{i=0}^{n-1}(1-\theta) f\left(Y_{i}\right)+h \sum_{i=0}^{n-1} \theta f\left(Y_{i+1}\right)+\sum_{i=0}^{n-1} \frac{1}{H+\frac{1}{2}}\left(t_{n}-t_{i}\right)^{H+\frac{1}{2}} g\left(Y_{i}\right) \Delta B_{i} .
$$

We devote to proving that the strong convergence order of this method is 1 .

This paper is organized as follows. In Section 2, we consider the well-posedness of analytical solutions of SVIDEs (1.1), moment boundedness and Hölder continuity. Section 3 shows the order of strong convergence of $\theta$-Maruyama method (1.3) is 1 . Numerical experiments are presented in the final section.

\section{Well-posedness of SVIDEs}

Throughout this paper, unless otherwise specified, we use the following notations. Let $E$ denote the expectation corresponding to $P$. Let $|\cdot|$ denote both the Euclidean norm on $\mathbb{R}^{d}$ and the trace (or Frobenius) norm on $\mathbb{R}^{d \times m}$. If $S$ is a set, then its indicator function is denoted by $\mathbb{1}_{S}$, namely $\mathbb{1}_{S}(x)=1$ if $x \in S$ and 0 otherwise.

In this section, we mainly discuss the well-posedness and moment boundedness of the analytic solutions of (1.1). In order to ensure the existence and uniqueness of the analytic solutions of (1.1) and study the strong convergence of its numerical method, we further assume that the drift term $f(x)$ and the diffusion term $g(x)$ satisfy the following conditions:

Assumption 2.1 (Global Lipschitz condition). There exists a positive constant $L$ such that for $\forall y, z \in \mathbb{R}^{d}$, the inequality

$$
|f(y)-f(z)| \vee|g(y)-g(z)| \leq L|y-z|
$$

holds, where and hereinafter $\vee$ denotes the largest of the two terms. 
Assumption 2.2 (Linear growth condition). There exists a positive constant $K$ such that for $\forall y \in \mathbb{R}^{d}$, the inequality

$$
|f(y)|^{2} \vee|g(y)|^{2} \leq K\left(1+|y|^{2}\right)
$$

holds.

Theorem 2.1. $x(t)$ is a solution of Eq. (1.1) if and only if it is a solution of Eq. (1.2).

Proof. Eq. (1.1) can be rewritten as

$$
x(t)=x(0)+\int_{0}^{t} f(x(s)) d s+\int_{0}^{t} \int_{0}^{s}(s-\tau)^{H-\frac{1}{2}} g(x(\tau)) d B(\tau) d s .
$$

The stochastic Fubini theorem [37] shows

$$
\begin{aligned}
x(t) & =x(0)+\int_{0}^{t} f(x(s)) d s+\int_{0}^{t} \int_{\tau}^{t}(s-\tau)^{H-\frac{1}{2}} g(x(\tau)) d s d B(\tau) \\
& =x(0)+\int_{0}^{t} f(x(s)) d s+\int_{0}^{t} \frac{1}{H+\frac{1}{2}}(t-s)^{H+\frac{1}{2}} g(x(s)) d B(s) .
\end{aligned}
$$

From the above, Eq. (1.2) is an equivalent form of (1.1), since the stochastic Fubini theorem is also true in reverse. Therefore, the proof is completed.

Now we prove the existence, uniqueness and boundedness of analytical solutions of the nonlinear SVIDEs (1.1).

Theorem 2.2. Let the Assumptions 2.1 and 2.2 hold. Then, there exists a unique strong solution $x(t)$ to (1.1). Moreover, $E|x(t)|^{2}<\infty$ for all $t \in[0, T]$.

Proof. According to Theorem 2.1, we define the operator $\Psi$ by

$$
\Psi x(t)=x(0)+\int_{0}^{t} f(x(s)) d s+\int_{0}^{t} \frac{1}{H+\frac{1}{2}}(t-s)^{H+\frac{1}{2}} g(x(s)) d B(s) .
$$

Then, by the elementary inequality, Hölder inequality as well as the Assumption 2.1, it holds that

$$
\begin{aligned}
& E\left|\Psi x_{1}(t)-\Psi x_{2}(t)\right|^{2} \\
\leq & 2 E\left|\int_{0}^{t}\left(f\left(x_{1}(s)\right)-f\left(x_{2}(s)\right)\right) d s\right|^{2}+2 E\left|\int_{0}^{t} \frac{1}{H+\frac{1}{2}}(t-s)^{H+\frac{1}{2}}\left(g\left(x_{1}(s)\right)-g\left(x_{2}(s)\right)\right) d B(s)\right|^{2} \\
\leq & 2 T \int_{0}^{t} E\left|f\left(x_{1}(s)\right)-f\left(x_{2}(s)\right)\right|^{2} d s+\frac{2}{\left(H+\frac{1}{2}\right)^{2}} \int_{0}^{t}(t-s)^{2 H+1} E\left|g\left(x_{1}(s)\right)-g\left(x_{2}(s)\right)\right|^{2} d s \\
\leq & 2 L^{2} T \int_{0}^{t} E\left|x_{1}(s)-x_{2}(s)\right|^{2} d s+\frac{2 L^{2}}{\left(H+\frac{1}{2}\right)^{2}} \int_{0}^{t}(t-s)^{2 H+1} E\left|x_{1}(s)-x_{2}(s)\right|^{2} d s \\
\leq & \left(2 L^{2} T+\frac{2 L^{2} T^{2 H+1}}{\left(H+\frac{1}{2}\right)^{2}}\right) \int_{0}^{t} E\left|x_{1}(s)-x_{2}(s)\right|^{2} d s .
\end{aligned}
$$


Now, we introduce a norm by

$$
\|x\|=\max _{t \in[0, T]}\left\{e^{-M t} E|x(t)|^{2}\right\},
$$

where

$$
M=2 L^{2} T+\frac{2 L^{2} T^{2 H+1}}{\left(H+\frac{1}{2}\right)^{2}} .
$$

Hence, we have

$$
\begin{aligned}
\left\|\Psi x_{1}-\Psi x_{2}\right\| & =\max _{t \in[0, T]}\left\{e^{-M t} E\left|\Psi x_{1}(t)-\Psi x_{2}(t)\right|^{2}\right\} \\
& \leq \max _{t \in[0, T]} e^{-M t} \int_{0}^{t} M E\left|x_{1}(s)-x_{2}(s)\right|^{2} d s \\
& =\max _{t \in[0, T]} e^{-M t} \int_{0}^{t} M e^{M s} e^{-M s} E\left|x_{1}(s)-x_{2}(s)\right|^{2} d s \\
& \leq \max _{t \in[0, T]} e^{-M t} \int_{0}^{t} M e^{M s} \max _{s \in[0, T]}\left(e^{-M s} E\left|x_{1}(s)-x_{2}(s)\right|^{2}\right) d s \\
& \leq \max _{t \in[0, T]} e^{-M t} \int_{0}^{t} M e^{M s} d s\left\|x_{1}-x_{2}\right\| \\
& =\max _{t \in[0, T]}\left(1-e^{-M t}\right)\left\|x_{1}-x_{2}\right\| .
\end{aligned}
$$

By contraction mapping principle, there exits a unique solution to (1.1). Then, let's prove the boundedness of $E|x(t)|^{2}$. Using the elementary inequality and Hölder inequality, we obtain

$$
\begin{aligned}
E|x(t)|^{2} & \leq 3 E|x(0)|^{2}+3 E\left|\int_{0}^{t} f(x(s)) d s\right|^{2}+3 E\left|\int_{0}^{t} \frac{1}{H+\frac{1}{2}}(t-s)^{H+\frac{1}{2}} g(x(s)) d B(s)\right|^{2} \\
& \leq 3 E|x(0)|^{2}+3 T \int_{0}^{t} E|f(x(s))|^{2} d s+3 \int_{0}^{t}\left(\frac{1}{H+\frac{1}{2}}\right)^{2}(t-s)^{2 H+1} E|g(x(s))|^{2} d s .
\end{aligned}
$$

Combining Assumption 2.2, we have

$$
\begin{aligned}
E|x(t)|^{2} & \leq 3 E|x(0)|^{2}+3 T K \int_{0}^{t} E\left(1+|x(s)|^{2}\right) d s+3 K \int_{0}^{t}\left(\frac{1}{H+\frac{1}{2}}\right)^{2}(t-s)^{2 H+1} E\left(1+|x(s)|^{2}\right) d s \\
& \leq 3 E|x(0)|^{2}+3 T^{2} K+3 T K \int_{0}^{t} E|x(s)|^{2} d s+3 K C T^{2 H+2}+3 K C T^{2 H+1} \int_{0}^{t} E|x(s)|^{2} d s \\
& =C_{1}+C_{2} \int_{0}^{t} E|x(s)|^{2} d s
\end{aligned}
$$

where

$$
C=\left(\frac{1}{H+\frac{1}{2}}\right)^{2}, \quad C_{1}=3 E|x(0)|^{2}+3 K T^{2}+3 K C T^{2 H+2}, \quad C_{2}=3 T K+3 C K T^{2 H+1} .
$$

Therefore, based on Gronwall inequality, the proof is completed. 
Theorem 2.3. Let $0 \leq t_{1}<t_{2} \leq T$ and the Assumptions 2.1 and 2.2 hold. Then there exists $a$ positive constant $C$ such that

$$
E\left|x\left(t_{1}\right)-x\left(t_{2}\right)\right|^{2} \leq C\left|t_{1}-t_{2}\right|^{2} .
$$

Proof. Based on Assumptions 2.1 and 2.2 as well as Gronwall inequality, the proof is similar to that of Theorem 2.2 in [19] and is omitted.

Remark 2.1. Theorem 2.3 reveals that the exact solutions of Eq. (1.1) are Lipschitz continuous in root mean square sense. Therefore, we can expect the numerical method (1.3) is strongly convergent of order 1 , which will be investigated in the next section in details.

\section{Strong convergence of $\theta$-Maruyama method}

In this section, we present the $\theta$-Maruyama method for (1.2), which will be proved to be strongly convergent with first order.

For positive integer $N$, let $\mathcal{I}_{N}=\left\{t_{n}=n \frac{T}{N}: n=0,1, \cdots, N\right\}$ be a given uniform mesh on $[0, T]$. When $t=t_{n}$, Eq. (1.2) can be written as

$$
\begin{aligned}
x\left(t_{n}\right)=x_{0} & +\int_{0}^{t_{n}} f(x(s)) d s+\int_{0}^{t_{n}} \frac{1}{H+\frac{1}{2}}\left(t_{n}-s\right)^{H+\frac{1}{2}} g(x(s)) d B(s) \\
=x_{0} & +\sum_{i=0}^{n-1} \int_{t_{i}}^{t_{i+1}} f(x(s)) d s+\sum_{i=0}^{n-1} \int_{t_{i}}^{t_{i+1}} \frac{1}{H+\frac{1}{2}}\left(t_{n}-s\right)^{H+\frac{1}{2}} g(x(s)) d B(s) \\
=x_{0} & +\sum_{i=0}^{n-1} \int_{t_{i}}^{t_{i+1}}(1-\theta) f(x(s)) d s+\sum_{i=0}^{n-1} \int_{t_{i}}^{t_{i+1}} \theta f(x(s)) d s \\
& +\sum_{i=0}^{n-1} \int_{t_{i}}^{t_{i+1}} \frac{1}{H+\frac{1}{2}}\left(t_{n}-s\right)^{H+\frac{1}{2}} g(x(s)) d B(s) \\
=x_{0} & +\sum_{i=0}^{n-1} \int_{t_{i}}^{t_{i+1}}(1-\theta) f\left(x\left(t_{i}\right)\right) d s+\sum_{i=0}^{n-1} \int_{t_{i}}^{t_{i+1}} \theta f\left(x\left(t_{i+1}\right)\right) d s \\
& +\sum_{i=0}^{n-1} \int_{t_{i}}^{t_{i+1}} \frac{1}{H+\frac{1}{2}}\left(t_{n}-t_{i}\right)^{H+\frac{1}{2}} g\left(x\left(t_{i}\right)\right) d B(s)+R_{N} .
\end{aligned}
$$

We remove the remaining item $R_{N}$, then define $Y_{0}=x_{0}$ and

$$
Y_{n}=x_{0}+h \sum_{i=0}^{n-1}(1-\theta) f\left(Y_{i}\right)+h \sum_{i=0}^{n-1} \theta f\left(Y_{i+1}\right)+\sum_{i=0}^{n-1} \frac{1}{H+\frac{1}{2}}\left(t_{n}-t_{i}\right)^{H+\frac{1}{2}} g\left(Y_{i}\right) \Delta B_{i},
$$

where $n=1, \cdots, N, \Delta B_{i}=B\left(t_{i+1}\right)-B\left(t_{i}\right)$ indicates the increment of Brownian motion. At $t \in[0, T]$, let

$$
\hat{Y}(t)=\sum_{n=0}^{N} Y_{n} \mathbb{1}_{\left[t_{n}, t_{n+1}\right)}, \quad \check{Y}(t)=\sum_{n=0}^{N} Y_{n+1} \mathbb{1}_{\left[t_{n}, t_{n+1}\right)},
$$


which are simple step processes. Therefore, the $\theta$-Maruyama method follows:

$$
Y(t)=x_{0}+\int_{0}^{t}(1-\theta) f(\hat{Y}(s)) d s+\int_{0}^{t} \theta f(\check{Y}(s)) d s+\int_{0}^{t} \frac{(t-\underline{s})^{H+\frac{1}{2}}}{H+\frac{1}{2}} g(\hat{Y}(s)) d B(s),
$$

where $t:=t_{n}$ for $t \in\left[t_{n}, t_{n+1}\right)$. Note that, $Y_{n}=Y\left(t_{n}\right)=\hat{Y}(t), Y_{n+1}=Y\left(t_{n+1}\right)=\check{Y}(t)$.

Remark 3.1. In fact, the method (3.2) is consistent with the explicit EM method (4) of [19] when $\theta=0$. In fact, if $\theta=0$, then the method (3.2) becomes

$$
\begin{aligned}
Y_{n+1}=Y_{n} & +h f\left(Y_{n}\right)+\sum_{i=0}^{n} \frac{1}{H+\frac{1}{2}}\left(t_{n+1}-t_{i}\right)^{H+\frac{1}{2}} g\left(Y_{i}\right) \Delta B_{i} \\
& \quad-\sum_{i=0}^{n-1} \frac{1}{H+\frac{1}{2}}\left(t_{n}-t_{i}\right)^{H+\frac{1}{2}} g\left(Y_{i}\right) \Delta B_{i} \\
=Y_{n} & +h f\left(Y_{n}\right)+\frac{1}{H+\frac{1}{2}}\left(t_{n+1}-t_{n}\right)^{H+\frac{1}{2}} g\left(Y_{n}\right) \Delta B_{n} \\
& +\sum_{i=0}^{n-1} \frac{1}{H+\frac{1}{2}}\left[\left(t_{n+1}-t_{i}\right)^{H+\frac{1}{2}}-\left(t_{n}-t_{i}\right)^{H+\frac{1}{2}}\right] g\left(Y_{i}\right) \Delta B_{i} .
\end{aligned}
$$

Note that

$$
\begin{aligned}
& \frac{1}{H+\frac{1}{2}}\left(\left(t_{n+1}-t_{i}\right)^{H+\frac{1}{2}}-\left(t_{n}-t_{i}\right)^{H+\frac{1}{2}}\right) \\
= & \frac{1}{H+\frac{1}{2}}\left(\left(t_{n+1}-t_{i}\right)^{H+\frac{1}{2}}-\left(t_{n+1}-t_{i+1}\right)^{H+\frac{1}{2}}\right) \\
= & \int_{t_{i}}^{t_{i+1}}\left(t_{n+1}-\tau\right)^{H-\frac{1}{2}} d \tau .
\end{aligned}
$$

Then, we have

$$
\begin{aligned}
Y_{n+1} & =Y_{n}+h f\left(Y_{n}\right)+\sum_{i=0}^{n} \int_{t_{i}}^{t_{i+1}}\left(t_{n+1}-\tau\right)^{H-\frac{1}{2}} d \tau g\left(Y_{i}\right) \Delta B_{i} \\
& =Y_{n}+h f\left(Y_{n}\right)+\sum_{i=0}^{n} \int_{t_{i}}^{t_{i+1}} \int_{t_{i}}^{t_{i+1}}\left(t_{n+1}-\tau\right)^{H-\frac{1}{2}} d \tau g\left(Y_{i}\right) d B(s) .
\end{aligned}
$$

Hence, the $\theta$-Maruyama method degenerates to the EM method in [19].

Theorem 3.1. Under the conditions of Assumptions 2.1 and 2.2, there exists a positive constant C such that

$$
E\left(|Y(t)|^{2}\right) \leq C, \quad E\left(|\hat{Y}(t)|^{2}\right) \leq C, \quad E\left(|\check{Y}(t)|^{2}\right) \leq C, \quad \forall t \in[0, T]
$$

Proof. The proof is similar to that of Theorem 2.1 and the detail of the proof is omitted. 
In order to estimate the error of $\theta$-Maruyama method, for arbitrary $t \in\left[t_{n}, t_{n+1}\right)$, it follows (3.2) and (3.3) that

$$
\begin{aligned}
Y(t)-Y\left(t_{n}\right)= & \int_{t_{n}}^{t} \\
& +\int_{0}^{t_{n}} \frac{(t-\underline{s})^{H+\frac{1}{2}}-\left(t_{n}-\underline{s}\right)^{H+\frac{1}{2}}}{H+\frac{1}{2}} g(\hat{Y}(s)) d B(s) \\
& +\int_{t_{n}}^{t} \frac{\left(t-t_{n}\right)^{H+\frac{1}{2}}}{H+\frac{1}{2}} g(\hat{Y}(s)) d B(s) \\
= & L_{1}+L_{2}+L_{3},
\end{aligned}
$$

where $L_{1}, L_{2}, L_{3}$ are defined by

$$
\begin{aligned}
L_{1} & :=\int_{t_{n}}^{t}(1-\theta) f(\hat{Y}(s)) d s+\int_{t_{n}}^{t} \theta f(\check{Y}(s)) d s, \\
L_{2} & :=\int_{0}^{t_{n}} \frac{(t-\underline{s})^{H+\frac{1}{2}}-\left(t_{n}-\underline{s}\right)^{H+\frac{1}{2}}}{H+\frac{1}{2}} g(\hat{Y}(s)) d B(s), \\
L_{3} & :=\int_{t_{n}}^{t} \frac{\left(t-t_{n}\right)^{H+\frac{1}{2}}}{H+\frac{1}{2}} g(\hat{Y}(s)) d B(s) .
\end{aligned}
$$

Lemma 3.1. If the Assumptions 2.1 and 2.2 hold. Then, there exists a positive constant $C$ such that

$$
\begin{aligned}
& E L_{1}^{2} \leq C h^{2}, \\
& E L_{2}^{2} \leq C h^{2}, \\
& E L_{3}^{2} \leq C h^{2 H+2} .
\end{aligned}
$$

Proof. For the estimate (3.7a), it follows from Hölder inequality, Theorem 3.1 and Assumption 2.2 that

$$
\begin{aligned}
E L_{1}^{2} & =E\left|\int_{t_{n}}^{t}(1-\theta) f(\hat{Y}(s)) d s+\int_{t_{n}}^{t} \theta f(\check{Y}(s)) d s\right|^{2} \\
& \leq 2 E\left|\int_{t_{n}}^{t}(1-\theta) f(\hat{Y}(s)) d s\right|^{2}+2 E\left|\int_{t_{n}}^{t} \theta f(\check{Y}(s)) d s\right|^{2} \\
& \leq 2\left(t-t_{n}\right)(1-\theta)^{2} E\left(\int_{t_{n}}^{t}|f(\hat{Y}(s))|^{2} d s\right)+2\left(t-t_{n}\right) \theta^{2} E\left(\int_{t_{n}}^{t}|f(\check{Y}(s))|^{2} d s\right) \\
& \leq 2\left(t-t_{n}\right)(1-\theta)^{2} K\left(\int_{t_{n}}^{t} E\left(1+|\hat{Y}(s)|^{2}\right) d s\right)+2\left(t-t_{n}\right) K \theta^{2}\left(\int_{t_{n}}^{t} E\left(1+|\check{Y}(s)|^{2}\right) d s\right) \\
& \leq C h^{2} .
\end{aligned}
$$


Then, for the estimate (3.7b)

$$
\begin{aligned}
E L_{2}^{2} & =E\left|\int_{0}^{t_{n}} \frac{(t-\underline{s})^{H+\frac{1}{2}}-\left(t_{n}-\underline{s}\right)^{H+\frac{1}{2}}}{H+\frac{1}{2}} g(\hat{Y}(s)) d B(s)\right|^{2} \\
& =E \int_{0}^{t_{n}}\left(\frac{(t-\underline{s})^{H+\frac{1}{2}}-\left(t_{n}-\underline{s}\right)^{H+\frac{1}{2}}}{H+\frac{1}{2}}\right)^{2}|g(\hat{Y}(s))|^{2} d s \\
& =E \sum_{i=0}^{n-1} \int_{t_{i}}^{t_{i+1}}\left(\frac{\left(t-t_{i}\right)^{H+\frac{1}{2}}-\left(t_{n}-t_{i}\right)^{H+\frac{1}{2}}}{H+\frac{1}{2}}\right)^{2}|g(\hat{Y}(s))|^{2} d s .
\end{aligned}
$$

Noting that the estimate

$$
\frac{\left(t-t_{i}\right)^{H+\frac{1}{2}}-\left(t_{n}-t_{i}\right)^{H+\frac{1}{2}}}{H+\frac{1}{2}}=\int_{t_{n}}^{t}\left(s-t_{i}\right)^{H-\frac{1}{2}} d s \leq\left(t-t_{n}\right)\left(t_{n}-t_{i}\right)^{H-\frac{1}{2}}
$$

holds. By combining the inequality (3.9) with Assumption 2.2 and Theorem 3.1, we have

$$
\begin{aligned}
E L_{2}^{2} & \leq C \sum_{i=0}^{n-1} \int_{t_{i}}^{t_{i+1}}\left(t-t_{n}\right)^{2}\left(t_{n}-t_{i}\right)^{2 H-1} E\left(1+|\hat{Y}(s)|^{2}\right) d s \\
& \leq C \sum_{i=0}^{n-1} \int_{t_{i}}^{t_{i+1}}\left(t-t_{n}\right)^{2}\left(t_{n}-t_{i}\right)^{2 H-1} d s \\
& \leq C h^{2+2 H} \sum_{i=0}^{n-1}(n-i)^{2 H-1} \leq C h^{2} .
\end{aligned}
$$

Finally, for the estimate (3.7c). In a similar manner, by Theorem 3.1 and Assumption 2.2, we can prove that

$$
\begin{aligned}
E L_{3}^{2} & =E\left|\int_{t_{n}}^{t} \frac{\left(t-t_{n}\right)^{H+\frac{1}{2}}}{H+\frac{1}{2}} g(\hat{Y}(s)) d B(s)\right|^{2} \\
& =E\left(\int_{t_{n}}^{t} \frac{\left(t-t_{n}\right)^{2 H+1}}{\left(H+\frac{1}{2}\right)^{2}}|g(\hat{Y}(s))|^{2} d s\right) \\
& \leq K \int_{t_{n}}^{t} \frac{\left(t-t_{n}\right)^{2 H+1}}{\left(H+\frac{1}{2}\right)^{2}} E\left(1+|\hat{Y}(s)|^{2}\right) d s \\
& \leq C \int_{t_{n}}^{t} \frac{\left(t-t_{n}\right)^{2 H+1}}{\left(H+\frac{1}{2}\right)^{2}} d s=\frac{C\left(t-t_{n}\right)^{2+2 H}}{\left(H+\frac{1}{2}\right)^{2}} \\
& \leq C h^{2 H+2} .
\end{aligned}
$$

Here the kernel function $\left(t-t_{n}\right)^{H+\frac{1}{2}}$ improves the order of the estimation (3.7c), which implies that the order of (3.7c) depends on $H$ and has the the form $h^{2+2 H}$ but not $h^{2}$. This completes the proof of this lemma. 
Theorem 3.2. If the Assumptions 2.1 and 2.2 are satisfied, then there is a constant $C$ such that

$$
E\left[|Y(t)-\hat{Y}(t)|^{2}\right] \vee E\left[|Y(t)-\check{Y}(t)|^{2}\right] \leq C h^{2}, \quad \forall t \in[0, T] .
$$

Proof. Using elementary inequality and Lemma 3.1, we get

$$
\begin{aligned}
E|Y(t)-\hat{Y}(t)|^{2} & \leq 3 E L_{1}^{2}+3 E L_{2}^{2}+3 E L_{3}^{2} \\
& \leq C h^{2}+C h^{2}+C h^{2 H+2} \\
& \leq C h^{2} .
\end{aligned}
$$

By the above conclusions and elementary inequality, a similar approach yields that

$$
\begin{aligned}
E|Y(t)-\check{Y}(t)|^{2} & =E|Y(t)-\hat{Y}(t)+\hat{Y}(t)-\check{Y}(t)|^{2} \\
& \leq 2 E|Y(t)-\hat{Y}(t)|^{2}+2 E|\check{Y}(t)-\hat{Y}(t)|^{2} \\
& \leq C h^{2}+E|\check{Y}(t)-\hat{Y}(t)|^{2} .
\end{aligned}
$$

For any $t \in[0, T]$, there exist a unique integer $n$ such that $t \in\left[t_{n}, t_{n+1}\right)$, and we can get

$$
\check{Y}(t)=Y\left(t_{n+1}\right), \quad \hat{Y}(t)=Y\left(t_{n}\right) .
$$

By replacing $t$ by $t_{n+1}$ in Lemma 3.1, this together with (3.13) implies

$$
E|\check{Y}(t)-\hat{Y}(t)|^{2}=E\left|Y\left(t_{n+1}\right)-Y\left(t_{n}\right)\right|^{2} \leq C h^{2} .
$$

Then we have

$$
E|Y(t)-\check{Y}(t)|^{2} \leq C h^{2}+E|\check{Y}(t)-\hat{Y}(t)|^{2} \leq C h^{2} .
$$

Summarizing the above results leads to the desired assertion.

Theorem 3.3. If the Assumptions 2.1 and 2.2 are satisfied, then there exists a constant $C$ such that

$$
E|x(t)-Y(t)|^{2} \leq C h^{2}, \quad \forall t \in[0, T] .
$$

Proof. According to (2.4) and (3.3), it holds that

$$
\begin{aligned}
x(t)-Y(t)=x_{0} & +\int_{0}^{t} f(x(s)) d s+\int_{0}^{t} \frac{(t-s)^{H+\frac{1}{2}}}{H+\frac{1}{2}} g(x(s)) d B(s) \\
& \quad-x_{0}-\int_{0}^{t}(1-\theta) f(\hat{Y}(s)) d s-\int_{0}^{t} \theta f(\check{Y}(s)) d s-\int_{0}^{t} \frac{(t-\underline{s})^{H+\frac{1}{2}}}{H+\frac{1}{2}} g(\hat{Y}(s)) d B(s) \\
= & \int_{0}^{t}(1-\theta)(f(x(s))-f(\hat{Y}(s))) d s+\int_{0}^{t} \theta(f(x(s))-f(\check{Y}(s))) d s \\
& +\int_{0}^{t} \frac{(t-s)^{H+\frac{1}{2}} g(x(s))-(t-\underline{s})^{H+\frac{1}{2}} g(\hat{Y}(s))}{H+\frac{1}{2}} d B(s) \\
= & J_{1}+J_{2}
\end{aligned}
$$


where

$$
\begin{aligned}
J_{1} & :=\int_{0}^{t}(1-\theta)(f(x(s))-f(\hat{Y}(s))) d s+\int_{0}^{t} \theta(f(x(s))-f(\check{Y}(s))) d s, \\
J_{2} & :=\int_{0}^{t} \frac{(t-s)^{H+\frac{1}{2}} g(x(s))-(t-\underline{s})^{H+\frac{1}{2}} g(\hat{Y}(s))}{H+\frac{1}{2}} d B(s) .
\end{aligned}
$$

Furthermore, it follows from elementary inequality, Hölder inequality, and Assumption 2.1 that

$$
\begin{aligned}
E J_{1}^{2} & =E\left|\int_{0}^{t}(1-\theta)(f(x(s))-f(\hat{Y}(s))) d s+\int_{0}^{t} \theta(f(x(s))-f(\check{Y}(s))) d s\right|^{2} \\
& \leq 2 E\left|\int_{0}^{t}(1-\theta)(f(x(s))-f(\hat{Y}(s))) d s\right|^{2}+2 E\left|\int_{0}^{t} \theta(f(x(s))-f(\check{Y}(s))) d s\right|^{2} \\
& \leq 2 T(1-\theta)^{2} \int_{0}^{t} E|f(x(s))-f(\hat{Y}(s))|^{2} d s+2 T \theta^{2} \int_{0}^{t} E|f(x(s))-f(\check{Y}(s))|^{2} d s \\
& \leq 2 T L^{2}(1-\theta)^{2} \int_{0}^{t} E|x(s)-\hat{Y}(s)|^{2} d s+2 T L^{2} \theta^{2} \int_{0}^{t} E|x(s)-\check{Y}(s)|^{2} d s .
\end{aligned}
$$

In a similar way, $J_{2}$ can be split as two terms again as follows:

$$
\begin{aligned}
J_{2}= & \int_{0}^{t} \frac{(t-s)^{H+\frac{1}{2}} g(x(s))-(t-\underline{s})^{H+\frac{1}{2}} g(\hat{Y}(s))}{H+\frac{1}{2}} d B(s) \\
= & \int_{0}^{t} \frac{(t-s)^{H+\frac{1}{2}}}{H+\frac{1}{2}}(g(x(s))-g(\hat{Y}(s))) d B(s) \\
& \quad+\int_{0}^{t} \frac{(t-s)^{H+\frac{1}{2}}-(t-\underline{s})^{H+\frac{1}{2}}}{H+\frac{1}{2}} g(\hat{Y}(s)) d B(s) \\
= & J_{21}+J_{22},
\end{aligned}
$$

where

$$
\begin{aligned}
& J_{21}:=\int_{0}^{t} \frac{(t-s)^{H+\frac{1}{2}}}{H+\frac{1}{2}}(g(x(s))-g(\hat{Y}(s))) d B(s), \\
& J_{22}:=\int_{0}^{t} \frac{(t-s)^{H+\frac{1}{2}}-(t-\underline{s})^{H+\frac{1}{2}}}{H+\frac{1}{2}} g(\hat{Y}(s)) d B(s) .
\end{aligned}
$$

Now, we give a sharp estimate for $J_{21}$

$$
\begin{aligned}
E J_{21}^{2} & =E\left|\int_{0}^{t} \frac{(t-s)^{H+\frac{1}{2}}}{H+\frac{1}{2}}(g(x(s))-g(\hat{Y}(s))) d B(s)\right|^{2} \\
& =\int_{0}^{t} \frac{(t-s)^{2 H+1}}{\left(H+\frac{1}{2}\right)^{2}} E|g(x(s))-g(\hat{Y}(s))|^{2} d s
\end{aligned}
$$




$$
\begin{aligned}
& \leq C \int_{0}^{t}(t-s)^{2 H+1} E|x(s)-\hat{Y}(s)|^{2} d s \\
& \leq T^{2 H+1} C \int_{0}^{t} E|x(s)-\hat{Y}(s)|^{2} d s,
\end{aligned}
$$

where Assumption 2.1 was used. Using Assumption 2.2 and Theorem 3.1, we get

$$
\begin{aligned}
E J_{22}^{2} & =E\left|\int_{0}^{t} \frac{(t-s)^{H+\frac{1}{2}}-(t-\underline{s})^{H+\frac{1}{2}}}{H+\frac{1}{2}} g(\hat{Y}(s)) d B(s)\right|^{2} \\
& \leq K \int_{0}^{t}\left[(t-s)^{H+\frac{1}{2}}-(t-\underline{s})^{H+\frac{1}{2}}\right]^{2} E\left(1+|\hat{Y}(s)|^{2}\right) d s \\
& \leq C \int_{0}^{t}\left[(t-s)^{H+\frac{1}{2}}-(t-\underline{s})^{H+\frac{1}{2}}\right]^{2} d s \\
& \leq C h^{2} .
\end{aligned}
$$

Thus,

$$
\begin{aligned}
& E|x(t)-Y(t)|^{2} \leq 2 E J_{1}^{2}+2 E J_{2}^{2} \\
\leq & 2\left(2 T L^{2}(1-\theta)^{2} \int_{0}^{t} E|x(s)-\hat{Y}(s)|^{2} d s+2 T L^{2} \theta^{2} \int_{0}^{t} E|x(s)-\check{Y}(s)|^{2} d s\right) \\
& \quad+2 T^{2 H+1} C \int_{0}^{t} E|x(s)-\hat{Y}(s)|^{2} d s+C h^{2} \\
\leq & C_{1} \int_{0}^{t} E|x(s)-\hat{Y}(s)|^{2} d s+C_{2} \int_{0}^{t} E|x(s)-\check{Y}(s)|^{2} d s+C h^{2} .
\end{aligned}
$$

By an analysis similar to the above, we obtain

$$
\begin{aligned}
& E|x(t)-Y(t)|^{2} \\
\leq & C_{1} \int_{0}^{t} E|x(s)-Y(s)+Y(s)-\hat{Y}(s)|^{2} d s+C_{2} \int_{0}^{t} E|x(s)-Y(s)+Y(s)-\check{Y}(s)|^{2} d s+C h^{2} \\
\leq & C_{1} \int_{0}^{t} 2 E|x(s)-Y(s)|^{2}+2 E|Y(s)-\hat{Y}(s)|^{2} d s+C_{2} \int_{0}^{t} 2 E|x(s)-Y(s)|^{2} \\
& \quad+2 E|Y(s)-\check{Y}(s)|^{2} d s+C h^{2} \\
\leq & 2\left(C_{1}+C_{2}\right) \int_{0}^{t} E|x(s)-Y(s)|^{2} d s+2\left(C_{1}+C_{2}\right) h^{2}+C h^{2}
\end{aligned}
$$

where elementary inequality and Theorem 3.2 were used. Thus,

$$
E|x(t)-Y(t)|^{2} \leq\left(2 C_{1}+2 C_{2}+C\right) h^{2} e^{\left(2 C_{1}+2 C_{2}\right) t}=C h^{2} .
$$

The final result follows from the Gronwall inequality.

Remark 3.2. Theorem 3.3 shows that the order of convergence of the $\theta$-Maruyama method is independent of $H$ and is 1 . This result actually improves Theorem 3.9 of [19] for the case of $H \in\left(0, \frac{1}{2}\right)$ based on Remark 3.10 in [19]. 
Remark 3.3. For all we know, the Assumptions 2.1 and 2.2 are all required so far for obtaining optimal convergence rates of numerical methods for stochastic Volterra integral equations whose diffusion terms are not semi-martingale [32]. It is difficult to relax the Assumptions 2.1 and 2.2 in order to get such optimal convergence rates.

\section{Numerical experiments}

In this section, we verify the convergence rate of the $\theta$-Maruyama method for the nonlinear SVIDEs (1.1). More precisely, we measure the mean-square errors at the terminal time $t_{N}$ by

$$
\varepsilon=\sqrt{\left.\frac{1}{5000} \sum_{i=1}^{5000} \mid X^{(i)}\left(t_{N}\right)-X_{N}^{(i)}\right)\left.\right|^{2}}
$$

where $X^{(i)}\left(t_{N}\right)$ and $X_{N}^{(i)}$ indicate respectively exact solutions and numerical solutions in the $i$ th sample path.

Since it is difficult to obtain explicitly the exact solution of Eq. (1.1), the numerical approximation of the $\theta$-Maruyama method with a small stepsize $h=2^{-12}$ is used as a replacement of the unknown exact solution. The numerical solutions of the $\theta$-Maruyama method and the corresponding errors $\varepsilon$ will be obtained by using seven different stepsizes $\Delta=2^{-2}, 2^{-3}, 2^{-4}, 2^{-5}, 2^{-6}, 2^{-7}, 2^{-8}$ on the same Brownian path.

Example 4.1. We consider the nonlinear SVIDEs

$$
\frac{d x(t)}{d t}=\sin (x)+\int_{0}^{t}(t-\tau)^{H-\frac{1}{2}} \cos (x) d B(\tau)
$$

with initial value $x(t)=1, t \in[0,1]$. The calculation results are shown in the tables below.

Table 1: Strong convergence orders of the $\theta$-Maruyama method with $\theta=0$.

\begin{tabular}{||cccccc||}
\hline$\Delta$ & $H=0.1$ & $H=0.3$ & $H=0.5$ & $H=0.8$ & $H=1.0$ \\
\hline $2^{-2}$ & $2.3625 \mathrm{e}-01$ & $1.6544 \mathrm{e}-01$ & $1.2471 \mathrm{e}-01$ & $9.8132 \mathrm{e}-02$ & $8.3628 \mathrm{e}-02$ \\
$2^{-3}$ & $1.2224 \mathrm{e}-01$ & $8.2389 \mathrm{e}-02$ & $6.1929 \mathrm{e}-02$ & $4.7464 \mathrm{e}-02$ & $4.0246 \mathrm{e}-02$ \\
$2^{-4}$ & $6.1498 \mathrm{e}-02$ & $4.0565 \mathrm{e}-02$ & $3.0449 \mathrm{e}-02$ & $2.2954 \mathrm{e}-02$ & $1.9633 \mathrm{e}-02$ \\
$2^{-5}$ & $3.1016 \mathrm{e}-02$ & $1.9804 \mathrm{e}-02$ & $1.4747 \mathrm{e}-02$ & $1.1015 \mathrm{e}-02$ & $9.4123 \mathrm{e}-03$ \\
$2^{-6}$ & $1.5045 \mathrm{e}-02$ & $9.3502 \mathrm{e}-03$ & $6.9566 \mathrm{e}-03$ & $5.2551 \mathrm{e}-03$ & $4.4475 \mathrm{e}-03$ \\
$2^{-7}$ & $6.7560 \mathrm{e}-03$ & $4.1103 \mathrm{e}-03$ & $3.0691 \mathrm{e}-03$ & $2.3369 \mathrm{e}-03$ & $1.9613 \mathrm{e}-03$ \\
$2^{-8}$ & $2.5725 \mathrm{e}-03$ & $1.5883 \mathrm{e}-03$ & $1.1562 \mathrm{e}-03$ & $8.5664 \mathrm{e}-04$ & $7.2223 \mathrm{e}-04$ \\
order & 1.0868 & 1.1171 & 1.1255 & 1.1400 & 1.1426 \\
\hline
\end{tabular}


Table 2: Strong convergence orders of the $\theta$-Maruyama method with $\theta=0.5$.

\begin{tabular}{||cccccc||}
\hline$\Delta$ & $H=0.1$ & $H=0.3$ & $H=0.5$ & $H=0.8$ & $H=1.0$ \\
\hline $2^{-2}$ & $2.0735 \mathrm{e}-01$ & $1.3484 \mathrm{e}-01$ & $1.0047 \mathrm{e}-01$ & $7.2795 \mathrm{e}-02$ & $6.2862 \mathrm{e}-02$ \\
$2^{-3}$ & $1.0663 \mathrm{e}-01$ & $6.7025 \mathrm{e}-02$ & $4.8549 \mathrm{e}-02$ & $3.5648 \mathrm{e}-02$ & $3.0008 \mathrm{e}-02$ \\
$2^{-4}$ & $5.3845 \mathrm{e}-02$ & $3.3205 \mathrm{e}-02$ & $2.4051 \mathrm{e}-02$ & $1.7520 \mathrm{e}-02$ & $1.5028 \mathrm{e}-02$ \\
$2^{-5}$ & $2.6531 \mathrm{e}-02$ & $1.6271 \mathrm{e}-02$ & $1.1678 \mathrm{e}-02$ & $8.5637 \mathrm{e}-03$ & $7.3191 \mathrm{e}-03$ \\
$2^{-6}$ & $1.2574 \mathrm{e}-02$ & $7.7489 \mathrm{e}-03$ & $5.5409 \mathrm{e}-03$ & $4.0895 \mathrm{e}-03$ & $3.4502 \mathrm{e}-03$ \\
$2^{-7}$ & $5.8406 \mathrm{e}-03$ & $3.5382 \mathrm{e}-03$ & $2.5333 \mathrm{e}-03$ & $1.8542 \mathrm{e}-03$ & $1.5728 \mathrm{e}-03$ \\
$2^{-8}$ & $2.2866 \mathrm{e}-03$ & $1.3522 \mathrm{e}-03$ & $9.5450 \mathrm{e}-04$ & $7.1510 \mathrm{e}-04$ & $6.0889 \mathrm{e}-04$ \\
order & 1.0838 & 1.1066 & 1.1196 & 1.1116 & 1.1150 \\
\hline
\end{tabular}

Table 3: Strong convergence orders of the $\theta$-Maruyama method with $\theta=1$.

\begin{tabular}{||cccccc||}
\hline$\Delta$ & $H=0.1$ & $H=0.3$ & $H=0.5$ & $H=0.8$ & $H=1.0$ \\
\hline $2^{-2}$ & $1.7952 \mathrm{e}-01$ & $1.0954 \mathrm{e}-01$ & $7.9887 \mathrm{e}-02$ & $5.6808 \mathrm{e}-02$ & $5.0556 \mathrm{e}-02$ \\
$2^{-3}$ & $9.2439 \mathrm{e}-02$ & $5.4939 \mathrm{e}-02$ & $3.7840 \mathrm{e}-02$ & $2.6963 \mathrm{e}-02$ & $2.2549 \mathrm{e}-02$ \\
$2^{-4}$ & $4.6559 \mathrm{e}-02$ & $2.7368 \mathrm{e}-02$ & $1.9210 \mathrm{e}-02$ & $1.3801 \mathrm{e}-02$ & $1.1699 \mathrm{e}-02$ \\
$2^{-5}$ & $2.3595 \mathrm{e}-02$ & $1.3506 \mathrm{e}-02$ & $9.5896 \mathrm{e}-03$ & $7.1104 \mathrm{e}-03$ & $6.0499 \mathrm{e}-03$ \\
$2^{-6}$ & $1.1316 \mathrm{e}-02$ & $6.5405 \mathrm{e}-03$ & $4.6192 \mathrm{e}-03$ & $3.4815 \mathrm{e}-03$ & $3.0191 \mathrm{e}-03$ \\
$2^{-7}$ & $5.2833 \mathrm{e}-03$ & $3.0274 \mathrm{e}-03$ & $2.1364 \mathrm{e}-03$ & $1.6193 \mathrm{e}-03$ & $1.4199 \mathrm{e}-03$ \\
$2^{-8}$ & $2.1721 \mathrm{e}-03$ & $1.2168 \mathrm{e}-03$ & $8.5970 \mathrm{e}-04$ & $6.5186 \mathrm{e}-04$ & $5.6900 \mathrm{e}-04$ \\
order & 1.0615 & 1.0820 & 1.0897 & 1.0742 & 1.0789 \\
\hline
\end{tabular}

\section{Conclusions}

Under the global Lipschitz condition and linear growth condition, this paper provides the existence-uniqueness theorem to the nonlinear SVIDEs driven by RiemannLiouville fractional Brownian motion by contraction mapping principle and proves the $\theta$-Maruyama method to be strongly convergent of order 1 , which can be also shown from the results in the above tables. For the convergence rate analysis of $\theta$-Maruyama method of (1.1), it is difficult to relax the global Lipschitz condition and linear growth condition to non-global Lipschitz condition and polynomial growth condition, respectively, which will be our next goal.

\section{Acknowledgements}

Deep thanks go to the referees for their many constructive comments and suggestions to improve this article. This research is supported by the National Natural Science Foundation of China (No. 12071403). 


\section{References}

[1] A. M. WAZWAZ, Linear and Nonlinear Integral Equations, Springer, 2011.

[2] A. S. V. MURThy AND J. G. VerWer, Solving parabolic integro-differential equations by an explicit integration method, J. Comput. Appl. Math., 39(1) (1992), pp. 121-132.

[3] R. K. MilleR, An integro-differential equation for rigid heat conductors with memory, J. Math. Anal. Appl., 66(2) (1978), pp. 313-332.

[4] M. E. GURTIN AND A. C. PIPKIN, A general theory of heat conduction with finite wave speeds, Arch. Ration Mech. Anal., 31(2) (1968), pp. 113-126.

[5] P. KLOEDEN AND A. NeUENKIRCH, Convergence of numerical methods for stochastic differential equations in mathematical finance, World Sci. Publ., 14 (2013), pp. 49-80.

[6] H. BRUNNER AND J. D. LAMBERT, Stability of numerical methods for Volterra integro-differential equations, Computing, 12(1) (1974), pp. 75-89.

[7] L. J. Shen, J. P. SHI AND J. T. Sun, Complete controllability of impulsive stochastic integrodifferential systems, Automatica, 46 (2010), pp. 1068-1073.

[8] Q. Y. LI AND S. Q. GAN, Mean-square exponential stability of stochastic theta methods for nonlinear stochastic delay integro-differential equations, J. Appl. Math. Comput., 39 (2012), pp. 69-87.

[9] S. Q. GAN, Dissipativity of the backward Euler method for nonlinear Volterra functional differential equations in Banach space, Adv. Differential Equations, 2015(1) (2015), pp. 1-9.

[10] J. A. BARDinA AND D. W. Allan, A statistical model of flicker noise, P. IEEE, 54 (1966), pp. 176-178.

[11] B. B. MANDELBROT AND J. W. VNESS, Fractional Brownian motions, fractional noises and applications, SIAM Rev., 10(4) (1968), pp. 422-437.

[12] J. L. HONG, C. Y. HuANG, K. MINOO AND X. WANG, Optimal strong convergence rate of a backward Euler type scheme for the Cox-Ingersoll-Ross model driven by fractional Brownian motion, Stoch. Process Appl., 130 (2020), pp. 2675-2692.

[13] F. C. KLEBANER, Introduction to Stochastic Calculus with Applications, Imperial College Press, 2005.

[14] J. BERAN, Statistics for Long-Memory Processes, Chapman Hall, 2017.

[15] E. E. PeTERS, Fractal Market Analysis: Applying Chaos Theory to Investment and Economics, John Wiley \& Sons, 1994.

[16] Y. Q. XIAO, Stochastic Equations Driven by Fractional Brownian Motion and Its Application in Option Pricing, Ph.D. Central South University, China, 2012.

[17] J. L. HONG, C. Y. HUANG AND X. WANG, Optimal rate of convergence for two classes of schemes to stochastic differential equations driven by fractional Brownian motions, IMA J. Numer. Anal., 41 (2021) pp. 1608-1638.

[18] X. Y. LI AND W. R. CAO, Numerical solution of stochastic delay differential equations driven by fractional brownian motion, Numer. Math. Theory Methods Appl., 39(4) (2017), pp. 289-315.

[19] Z. W. YANG, H. Z. YANG AND Z. C. YAO, Strong convergence analysis for Volterra integrodifferential equations with fractional Brownian motions, J. Comput. Appl. Math., 383 (2021), 113156.

[20] X. J. DAI, W. P. BU AND A. G. XIAO, Well-posedness and EM approximations for non-Lipschitz stochastic fractional integro-differential equations, J. Comput. Appl. Math., 356 (2019), pp. 377390.

[21] J. CUI AND N. N. BI, Averaging principle for neutral stochastic functional differential equations with impulses and non-Lipschitz coefficients, Stat. Probab. Lett., 163 (2020), 108775.

[22] T. D. VIET, Y. SHEHU AND O. S. IYIOLA, Weak and strong convergence theorems for solving 
pseudo-monotone variational inequalities with non-Lipschitz mappings, Numer. Algorithms, 84 (2020), pp. 795-823.

[23] M. LI, C. M. HuANG AND Y. Z. HU, Numerical methods for stochastic Volterra integral equations with weakly singular kernels, (2020), arXiv: 2004.04916v1.

[24] X. T. WANG, The Theory and Related Problems of Backward Stochastic Volterra Integral Equations, Ph.D. Shandong University, China, 2013.

[25] S. REZAPOUR AND S. H. ZAKERI, On weak and strong convergence results for generalized equilibrium variational inclusion problems in Hilbert spaces, Adv. Differential. Equations, 2020 (2020), 462.

[26] H. Z. YANG, Z. W. YANG AND S. F. MA, Theoretical and numerical analysis for Volterra integrodifferential equations with Itô integral under polynomially growth conditions, Appl. Math. Comput., 360 (2019), pp. 70-82.

[27] W. ZHANG, Convergence of the balanced Euler method for a class of stochastic Volterra integrodifferential equations with non-globally Lipschitz continuous coefficients, Appl. Numer. Math., 154 (2020), pp. 17-35.

[28] W. ZHANG, H. LiANG AND J. F. GAO, Theoretical and numerical analysis of the EulerMaruyama method for generalized stochastic Volterra integro-differential equations, Comput. Appl. Math., 365 (2020), 112364.

[29] W. ZHANG, Theoretical and numerical analysis of a class of stochastic Volterra integro-differential equations with non-globally Lipschitz continuous coefficients, Appl. Numer. Math., 147 (2020), pp. 254-276.

[30] H. LIANG, Z. W. YANG AND J. F. GAO, Strong superconvergence of the Euler-Maruyama method for linear stochastic Volterra integral equations, J. Comput. Appl. Math., 317 (2017), pp. 447-457.

[31] M. LI, C. M. HuANG AND P. HU, Mean-square stability and convergence of a split-step theta method for stochastic Volterra integral equations, J. Comput. Appl. Math., 382 (2021), 113077.

[32] J. F. GAO, H. LiANG AND S. F. MA, Strong convergence of the semi-implicit Euler method for nonlinear stochastic Volterra integral equations with constant delay, Appl. Math. Comput., 348 (2019), pp. 385-398.

[33] A. D. KHAlaf, M. AbouAgwa And A. MustafA, Stochastic Volterra integral equations with jumps and the strong superconvergence of the Euler-Maruyama approximation, J. Comput. Appl. Math., 382 (2020), 113071.

[34] X. J. DAI AND A. G. XIAO, Lévy-driven stochastic Volterra integral equations with doubly singular kernels: existence, uniqueness, and a fast EM method, Adv. Comput. Math., 46(5) (2020), pp. 803-845.

[35] M. LI, C. M. HUANG AND Y. Z. HU, Asymptotic separation for stochastic Volterra integral equations with doubly singular kernels, Appl. Math. Lett., 113 (2021), 106880.

[36] T. S. DoAn, P. T. HuOnG, P. E. Kloeden AND A. M. Vu, Euler-Maruyama scheme for Caputo stochastic fractional differential equations, J. Comput. Appl. Math., 380 (2020), 112989.

[37] G. D. PRATO AND J. ZABCZYK, Stochastic Equations in Infinite Dimensions, Cambridge University Press, 2014. 